\title{
Do current incretin mimetics exploit the full therapeutic potential inherent in GLP-1 receptor stimulation?
}

\author{
M. A. Nauck • O. Baranov • R. A. Ritzel • J. J. Meier
}

Received: 5 February 2013 / Accepted: 14 May 2013 /Published online: 8 June 2013

(C) Springer-Verlag Berlin Heidelberg 2013

\begin{abstract}
Glucagon-like peptide-1 (GLP-1) receptor agonists (RAs) are incretin-derived glucose-lowering agents that have been used for the treatment of type 2 diabetes since 2007. Agents such as exenatide (short-acting and once weekly preparations), liraglutide, taspoglutide, albiglutide and lixisenatide lower fasting glucose and $\mathrm{HbA}_{1 \mathrm{c}}$ upon subcutaneous injection, leading to glycaemic control that is equivalent to, or better than, that observed with other oral glucose-lowering agents or bedtime insulin. However, varying proportions of patients report nausea and vomiting, adverse events that typically narrow the therapeutic dose range. Furthermore, GLP-1 RAs reduce fasting glucose to a clinically meaningful extent, but not into the normal range. In contrast, where GLP-1 is administered as a short-term intravenous infusion, a full normalisation of glucose concentrations (approximately $5 \mathrm{mmol} / \mathrm{l}$ ) has been observed without any risk of gastrointestinal side effects. Subcutaneous infusions or injections of GLP-1 are much less effective. The present analysis relates the proportion of patients who report nausea following treatment with GLP-1 and GLP-1 RAs to the clinical effectiveness of the treatment (represented by the fasting glucose concentration achieved with treatment). The results suggest that GLP-1
\end{abstract}

Electronic supplementary material The online version of this article (doi:10.1007/s00125-013-2953-6) contains peer-reviewed but unedited supplementary material, which is available to authorised users.

M. A. Nauck $(\bowtie) \cdot$ O. Baranov

Diabeteszentrum Bad Lauterberg, Kirchberg 21,

37431 Bad Lauterberg im Harz, Germany

e-mail: nauck@diabeteszentrum.de

R. A. Ritzel

Department of Medicine, Division of Endocrinology, Diabetes and

Nuclear Medicine, Klinikum Schwabing, Munich, Germany

J. J. Meier

Division of Diabetology and Gastrointestinal Endocrinology,

St Josef-Hospital, Ruhr University, Bochum, Germany
RAs injected into the subcutaneous compartment do not exploit the full potential inherent in GLP-1 receptor activation. Reasons for this may include modifications of the peptide molecules in the subcutaneous environment or high local concentrations triggering side effects through GLP-1 receptors on autonomic nerves in subcutaneous adipose tissue. Elucidation of the mechanisms underlying differential responses to GLP-1/GLP-1 RAs administered intravenously vs subcutaneously may help to develop improved agents or modes of administration that are more effective and have fewer side effects.

Keywords Autonomic nervous system - Gastrointestinal adverse events · Glucagon-like peptide-1 (GLP-1) ·

Glucagon-like peptide-1 (GLP-1) receptor agonists · Incretin mimetics $\cdot$ Subcutaneous adipose tissue
Abbreviations
DPP-4 Dipeptidyl peptidase-4
GLP-1 Glucagon-like peptide-1
RA Receptor agonists

\section{GLP-1: a parent compound for novel incretin-based glucose-lowering drugs}

Glucagon-like peptide-1 (GLP-1) has attracted attention because of its ability to stimulate insulin secretion in a glucose-dependent manner, to suppress glucagon release when glucose concentrations are not in the hypoglycaemic range, and to elicit a number of extra-pancreatic effects (on appetite/satiety, gastrointestinal motility and the cardiovascular system) [1]. This spectrum of biological activities addresses important deficits typical for patients with type 2 diabetes (impaired beta cell function, hyperglucagonaemia, overeating/obesity, excessive postprandial rises in glucose 
and triacylglycerol concentrations, etc.). Furthermore, in proof-of-concept experiments, a short-term intravenous infusion (lasting 4 or more hours), has lowered glucose concentrations in previously hyperglycaemic patients into the normal fasting glucose concentration range [2-6]. This is consistent for patients at all stages of type 2 diabetes, including those with a long duration of the disease and receiving insulin treatment after well-documented (secondary) failure of treatment with oral antihyperglycaemic agents [4]. Glucose concentrations between 4 and $6.5 \mathrm{mmol} / \mathrm{l}$ were achieved in the majority of fasting patients, and after meals, glycaemic excursions were not higher than in non-diabetic control participants [7]. Typically, this is achieved at GLP-1 doses of around 1-1.2 $\mathrm{pmol} \mathrm{kg}^{-1} \mathrm{~min}^{-1}$ or even lower [6]. Under such circumstances, steady-state plasma levels of total GLP-1 (full-length sequences 7-36 amide or 7-37, plus dipeptidyl peptidase (DPP-4)-degraded sequences 9-36 amide and 9-37, plus minor forms produced by other peptidases) of around 60-150 pmol/1 correspond to steady-state plasma concentrations of intact, biologically active GLP-1 (not degraded/inactivated by DPP-4 or other enzymes) of approximately 10-15 pmol/1 [2-6]. Of note, almost no adverse events were reported; in other words, the doseresponse relationship allows for a full normalisation of glycaemia without triggering side effects such as nausea and other gastrointestinal upsets. Some relevant patient characteristics of the pertinent clinical studies are shown in electronic supplementary material (ESM) Table 1. Based on such studies, it was concluded that GLP-1 is a promising agent to treat hyperglycaemia in patients with type 2 diabetes, but that this would require continuous administration, since the circulation half-life of intact GLP-1 is between 1 and $2 \min [8,9]$.

GLP-1 injected subcutaneously Following the successful treatment of hyperglycaemia with intravenous GLP-1, the subcutaneous route was explored using GLP-1 bolus injections. Based on the dose range given above for intravenous GLP-1, approximately $0.3 \mathrm{nmol} / \mathrm{kg}$ body weight infused over $4 \mathrm{~h}$ can be employed successfully to normalise glycaemia; however, subcutaneous bolus administration of doses up to $1.5 \mathrm{nmol} / \mathrm{kg}$ had some effect, but fell short of being able to fully normalise fasting glucose concentrations [10]. In part, this may have been due to the short-lived peak in GLP-1 plasma concentrations, lasting no longer than 60-90 min, even when transiently high GLP-1 plasma concentrations were reached (far above the concentration range described for intravenous infusions [see above]). Furthermore, at doses of $0.5 \mathrm{nmol} / \mathrm{kg}$ or higher, impaired wellbeing, nausea and vomiting were observed in a dosedependent manner in up to $50 \%$ of participants (both diabetic and healthy) $[10,11]$. Based on these results, a lower bioavailability of GLP-1 has to be suspected when the peptide is delivered into the subcutaneous compartment vs intravenous administration (approximately $20 \%$ or even lower; references in ESM Tables 1 and 2); more strikingly, the dose-response relationships for normalising glycaemia and for eliciting severe side effects suggest that nausea and vomiting are observed at lower GLP-1 doses and plasma concentrations than are necessary to display the full glucose-lowering effect. With the pharmacokinetic profile of GLP-1 after subcutaneous bolus injection [10, 11], this may be the consequence of: (1) not achieving a sufficiently long period with sufficient elevations in GLP-1 plasma levels; and (2) reaching a very high plasma concentration peak, albeit for a short time, which is nevertheless sufficient to trigger adverse events (nausea and vomiting were described to coincide with GLP-1 concentration peaks).

Continuous subcutaneous GLP-1 infusions Assuming that the above-mentioned problems could potentially be circumvented by the continuous infusion rather than intermittent bolus administration of GLP-1, several studies have explored the effects of continuous subcutaneous infusions of GLP-1 at doses of 2.4 or $4.8 \mathrm{pmol} \mathrm{kg}^{-1} \mathrm{~min}^{-1}$. These doses were chosen approximately two- to fivefold higher than the doses typically used for intravenous administration, in order to compensate for the lower bioavailability (see above) [12-15]. Steady-state plasma concentrations of total GLP-1 reached with these infusions were $127 \pm 22 \mathrm{pmol} / \mathrm{l}$ [12], $108 \pm 8 \mathrm{pmol} /[13], 183 \pm 22$ [15] and $197 \pm 28 \mathrm{pmol} / \mathrm{l}$ after 1 week and $282 \pm 57 \mathrm{pmol} / \mathrm{l}$ after 6 weeks [14]. The respective levels of intact, biologically active GLP-1 were $23 \pm 4 \mathrm{pmol} / 1$ [12] or $35 \pm 3 \mathrm{pmol} / 1$ [13] (summarised in ESM Table 2). Even though these concentrations were strikingly similar to those produced by 'clinically effective' doses of intravenous GLP-1 [2-6], the glucoselowering effects were still much inferior. Thus, from baseline fasting glucose concentrations of 10-13 mmol/1 (comparable for studies with intravenous and subcutaneous GLP-1), the glucose levels reached with subcutaneous GLP-1 were between 10 and $11 \mathrm{mmol} / 1$ [12-15], compared with 5-6 mmol/1 for intravenous GLP-1 [2, 7]. Furthermore, with the doses employed in the intravenous experiments, very few episodes of nausea or vomiting have been reported. Therefore, two potential conclusions come to mind: (1) the fourfold excess dose of subcutaneously administered GLP-1 is still not sufficient to compensate for the reduced bioavailability compared with intravenously administered GLP-1. In other words, even higher doses of subcutaneous GLP-1 would be required in order to elicit the full therapeutic effect leading to a normalisation in glycaemia; (2) the dose-response relationships for intravenously and subcutaneously administered GLP-1 differ from each other. The latter hypothesis is supported by the fact that the glucose-lowering effects of intravenously and subcutaneously administered GLP-1 were 
considerably different, even when roughly similar steady-state GLP-1 concentrations were reported for both total and intact bioactive GLP-1 under both conditions (ESM Table 2).

Comparison of glucose-lowering effects of GLP-1 administered via the intravenous or subcutaneous routes Figure 1 depicts the fasting glucose concentrations achieved with GLP-1 (7-36 amide or 7-37) in relation to the percentage of patients reporting nausea with intravenous, subcutaneous bolus or continuous subcutaneous administration during published trials (for details, see ESM Table 1). Plasma concentrations (steady-state or peak) increased in proportion to the doses administered. In the case of intravenous GLP-1 administered to hyperglycaemic patients with type 2 diabetes, even small doses of GLP-1 generally normalised glucose concentrations without inducing gastrointestinal side effects. In contrast, subcutaneous bolus administration of GLP-1 frequently led to nausea and vomiting, but did not normalise levels of glycaemia unless administering two bolus injections $2 \mathrm{~h}$ apart [10]. Achieving a reduction or even normalisation of fasting glucose concentrations with a single instead of two sequential administrations would certainly have required even higher doses, which would be likely to trigger more adverse events. Finally, continuous subcutaneous administration of GLP-1 neither caused nausea (at plasma concentrations much lower than those achieved with bolus administration), nor fully normalised glucose concentrations [12-15].

As a caveat to this comparison, the unequal durations of GLP-1 exposure in different studies must be borne in mind: the majority of experiments employing intravenous GLP-1 have been carried out over 4-8 h, whereas studies using subcutaneous delivery were typically longer in duration. Thus, some wearing-off of the glucose-lowering efficacy of GLP-1 over time cannot be excluded as a factor contributing to these differences.

\section{Exenatide}

The first GLP-1 receptor agonist (RA; also referred to as an 'incretin mimetic') approved for the treatment of type 2 diabetes was exendin-4, a peptide from the saliva of the Gila lizard (Heloderma suspectum), which shares a high degree of sequence similarity with human GLP-1 and has the ability to stimulate the GLP-1 receptor [1]. Its synthetic form, exenatide, has a half-life of approximately $3.5 \mathrm{~h}$ in humans [16]. A single subcutaneous injection leads to elevations in plasma exenatide concentrations, peaking at approximately 90-120 min, and lasting for $\sim 6-8 \mathrm{~h}$ in total, making twicedaily injections necessary for the clinical treatment of type 2 diabetes [17]. Tolerability of increasing doses of exenatide is limited by side effects such as nausea and vomiting [18]. Slow up-titration mitigates some of these gastrointestinal problems and reduces the frequency and intensity of side effects [19]. Nevertheless, in clinical trials, the dose range that could be examined in longer-term studies was limited by unacceptable rates of nausea. Thus, the established dose regimens are a compromise and do not lead to a full normalisation of fasting glucose concentration, while always being associated with a certain degree of nausea [20,21].

Interestingly, highly effective doses of exenatide (stimulating first-phase insulin secretion in type 2-diabetic patients) administered as a continuous intravenous infusion did not lead to nausea (at steady-state concentrations of exenatide of $134 \mathrm{pg} / \mathrm{ml}$, equivalent to $32 \mathrm{pmol} / 1$ [molecular mass 4,186.6 Da]) [22]. This is to be considered a high concentration, since exenatide is not degraded by DPP-4

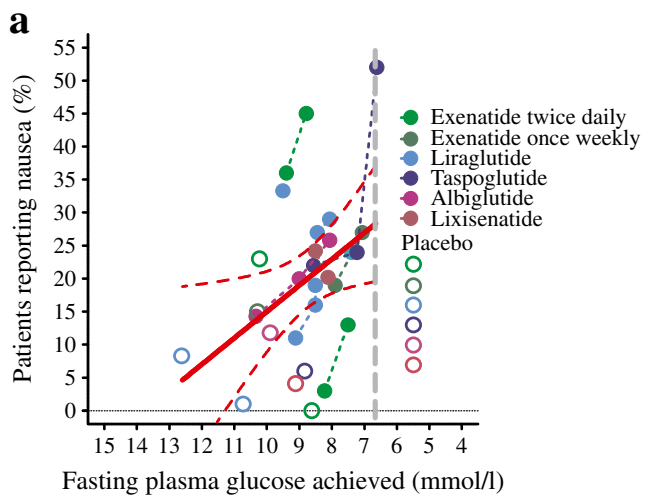

Fig. 1 Regression analysis relating the proportion of patients reporting nausea to clinical effectiveness in reducing glucose concentrations (as represented by the fasting plasma glucose reached): (a) in response to treatment with various GLP-1 RAs administered subcutaneously, (clinical trials); regression line: $y=54.8-0.22 x, r^{2}=0.165, p=0.029$; (b) after administration of GLP-1 through the subcutaneous route, regression line: $y=58.8-0.26 x, r^{2}=0.372, p=0.016$; (c) after administration
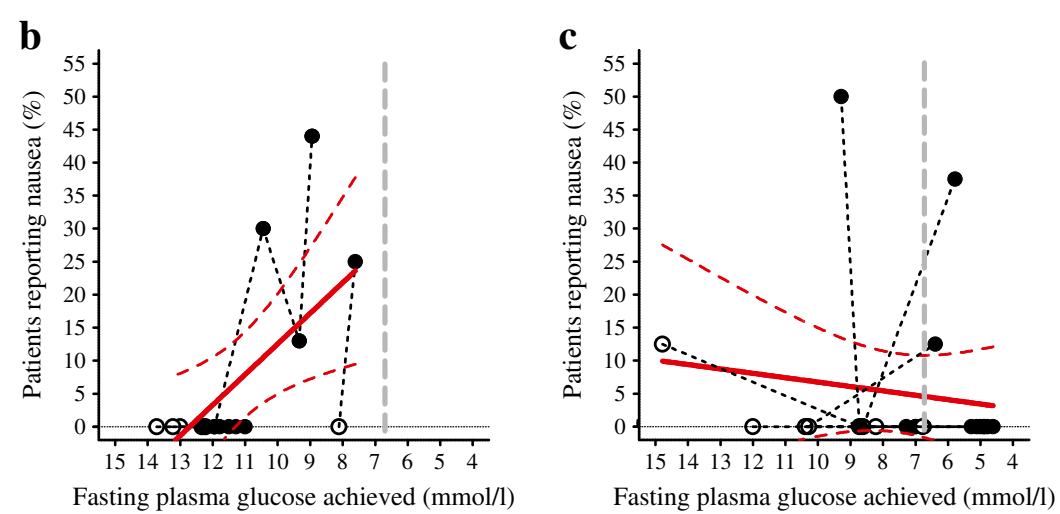

of GLP-1 through the intravenous route, regression line: $y=0.127+$ $0.037 x, r^{2}=0.018, p=0.55 ; 95 \%$ CIs are shown as dashed red lines around the regression (solid red lines). Filled symbols indicate results after administration of GLP-1 or GLP-1 RAs, open symbols indicate results after placebo administration. Symbols connected by lines were from the same study (different doses). Details of studies used for this analysis are listed in ESM Table 1 
and the level thus needs to be compared with intact GLP-1 plasma concentrations (ESM Table 2). However, in these experiments the efficiency variable measured was insulin secretory response, rather than the normalisation of glucose concentration [22]. On the other hand, in a study in which exenatide was administered as a continuous subcutaneous infusion, glycaemia was lowered significantly, although glucose concentrations remained well above truly normal levels, while at the same time nausea was reported by a high proportion of participating type 2-diabetic volunteers [23].

Taken together, the efficiency of exenatide as a glucosenormalising agent appears to be greater with intravenous infusions of the agent than with subcutaneous administration (bolus injection and continuous infusion) while the potential to induce gastrointestinal side effects is more pronounced with subcutaneous administrations. This mirrors the experiences with native GLP-1. While the ability to normalise fasting glycaemia with subcutaneous injections of exenatide may be limited by its relatively short half-life, which does not allow for effective drug levels to persist long enough after a pre-dinner injection to reduce fasting hyperglycaemia the next morning, this reasoning should not apply to longer acting GLP-1 RAs that, after repeated administration, will lead to a steady elevation in plasma concentration, with little variation over a typical $24 \mathrm{~h}$ period, including the overnight fasting period. Liraglutide was the first incretin mimetic developed with such properties.

\section{Liraglutide}

For single doses of liraglutide injected subcutaneously, the dose-limiting side effects are also nausea and/or vomiting [24]. Consistent with results from trials with exenatide, tolerability can be improved by slowly up-titrating the dose when initiating treatment with once-daily dosing [25, 26]. Nevertheless, while higher doses can be tolerated, the appropriate dose regimens used when starting liraglutide treatment give rise to a significantly more pronounced reduction in fasting glucose concentration than that seen with exenatide treatment [27], although this still falls short of the normalisation of glycaemia regularly observed with intravenous infusions of GLP-1. No studies have yet been reported on the use of intravenous or continuous subcutaneous liraglutide, which would help to test for similarities with conditions using GLP-1 or exenatide.

\section{Exploiting the full glucose-lowering potential of native GLP-1}

As a general conclusion from the comparisons detailed above, only GLP-1 and, potentially, exenatide administered intravenously appear to exploit the full glucose-lowering potential inherent in the mechanism of enhanced GLP-1 receptor stimulation. Briefly, this potential is summarised as the ability to fully normalise fasting glucose concentrations in patients with type 2 diabetes, irrespective of the duration of diabetes and their previous antihyperglycaemic treatment regimen. In contrast, subcutaneous administration, which has been used in proof-of-concept studies with GLP-1 and in numerous clinical trials involving various GLP-1 RAs, does not fully exploit this glucose-lowering potential: the bioavailability of GLP-1 and, probably, of other agents stimulating the GLP-1 receptor is substantially lower when injected into subcutaneous adipose tissue (see studies listed in ESM Table 1). This is obvious from the different doses of GLP-1 that have typically been administered through the intravenous (e.g. $1.2 \mathrm{pmol} \mathrm{kg}^{-1} \mathrm{~min}^{-1}$ ) vs the subcutaneous route (e.g. 2.4 or $4.8 \mathrm{pmol} \mathrm{kg}^{-1} \mathrm{~min}^{-1}$ or higher; ESM Table 1) and the plasma concentrations of total and intact GLP-1 that were reached with these regimens (ESM Table 2). Furthermore, subcutaneous administration of GLP-1 and GLP-1 RAs can result in adverse events such nausea, vomiting and other gastrointestinal side effects at doses that are too low to support a full normalisation of fasting glucose concentration in patients with type 2 diabetes (Fig. 1a, b). Thus, it is likely that all known GLP-1 RAs, including exenatide (both un-retarded and long-acting release once weekly preparations), liraglutide, and more novel agents such as taspoglutide, albiglutide and lixisenatide, which have also been developed for subcutaneous administration, do not fully exploit the potential inherent in this therapeutic principle. Figure 2 illustrates the hypothetical relationship between glucose-lowering effectiveness and the likelihood of triggering adverse events such as nausea and vomiting when GLP-1 (or GLP-1 RAs) are administered via the intravenous or subcutaneous route.

What might be the mechanism underlying this attenuated response to subcutaneous vs intravenous GLP-1? First, it is puzzling that studies with intravenous and subcutaneous administrations of GLP-1 reported similar circulating steady-state plasma concentrations for both total and intact GLP-1 (ESM Table 2), while the degree of normalisation in fasting glucose was significantly different (Fig. 1). This may point to some molecular modification of the GLP-1 molecule occurring while it is exposed to the subcutaneous environment. This potential change in structure might not be picked up by assays that are typically used to measure total (non-specific antibody) and intact (sandwich assay with two antibodies typically directed against both the $\mathrm{N}$-terminus and carboxy-terminus) GLP-1, because concentrations during intravenous and subcutaneous administration have been reported to be similar, although biological effects were clearly different (ESM Table 2). 

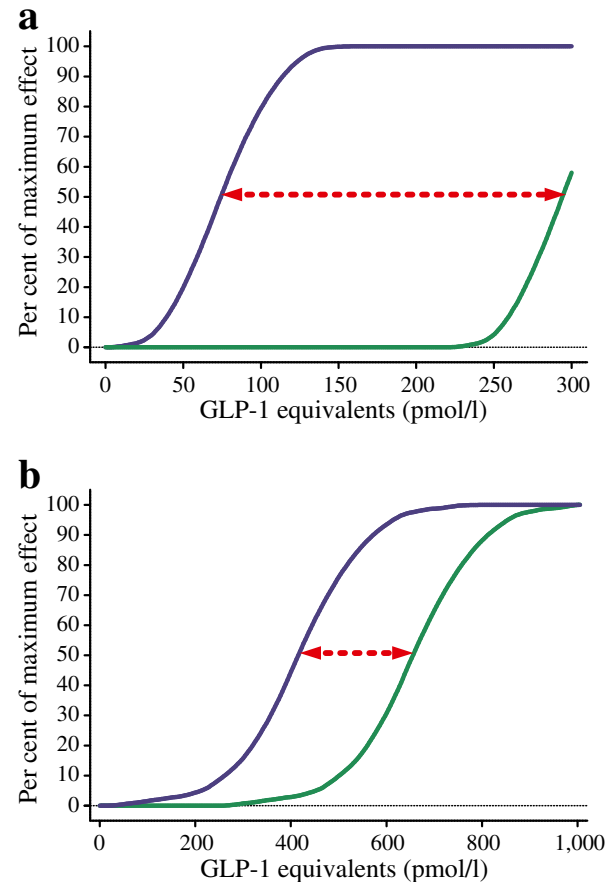

Fig. 2 Hypothetical dose-response relationships for plasma GLP-1 concentrations (or GLP-1 RA concentrations) vs glucose-lowering effects (blue lines) and the proportion of participants experiencing nausea (green lines) after (a) intravenous administration of GLP-1 and (b) subcutaneous administration of GLP-1 (or GLP-1 RAs). The double-sided red arrow indicates the therapeutic width under both conditions

A second possible explanation may be an interaction between high local concentrations of GLP-1 (or of related incretin mimetics) and GLP-1 receptors in adipose tissue. Adipose tissue has been found to be innervated by the autonomic nervous system, including peptidergic branches [28], and further study needs to be done on whether some of these nerves are equipped with GLP-1 receptors. In other regions, such as the gastrointestinal tract, nerve endings of the parasympathetic nervous system are rich in GLP-1 receptors, and interaction between GLP-1 and these nerves seems to mediate systemic responses, including profound changes in gastrointestinal motility [29, 30]. It is therefore hypothesised that exposure of GLP-1 receptors on nerve endings in the adipose tissue to high local concentrations of GLP-1 can potentially trigger side effects such as nausea and vomiting. If this occurs at doses that are not sufficient to elevate systemic concentrations of GLP-1 or GLP1-RAs into the truly therapeutic range, this could explain both the side effects and the lack of full glucose-lowering efficiency.

High GLP-1 concentrations in a similar range to those observed after the administration of long-acting GLP-1 RAs have been described in patients following gastric bypass surgery for obesity and type 2 diabetes [31-33], without a similar profile of adverse events; this fact seems to suggest that circulating concentrations alone are not responsible for inducing gastrointestinal side effects, since the same concentrations are easily tolerated after bariatric surgery (i.e. without a subcutaneous depot with excessively high local concentrations of GLP-1 RA). However, the anatomy of the gastrointestinal tract is changed considerably during bariatric surgery, and thus the anatomical substrate for eliciting gastrointestinal side effects may be different under these conditions, even if such adverse events are thought to be triggered by a direct interaction with the central nervous system.

Both theories, if confirmed by experimental studies, could lead to a substantial improvement in incretin-based therapeutic agents: if a molecular modification occurs, peptides resistant to these modifications could be developed, or the enzymes responsible could be inhibited by appropriate agents. If subcutaneous nerves mediate adverse gastrointestinal events, other routes of administration may promise more long-term success, and the subcutaneous administration of such agents may prove to be a dead end in terms of the potential for further improvements in GLP-1 RAs. We suggest that the obstacles associated with the subcutaneous administration of GLP-1 and incretin mimetics are worthy of more detailed study, as this will open new ways to circumvent problems that at the moment can only be roughly identified and described.

Acknowledgements We thank U. Buss, study nurse at the Diabeteszentrum Bad Lauterberg, for retrieving literature and for secretarial assistance.

Funding There has been no specific funding for the present analysis.

Duality of interest MAN has received research grants (to his institution, the Diabeteszentrum Bad Lauterberg) from Berlin-Chemie/Menarini, Eli Lilly \& Co., Merck, Sharp \& Dohme, Novartis Pharma, AstraZeneca, Boehringer Ingelheim, GlaxoSmithKline, MetaCure, Roche Pharma, Novo Nordisk Pharma and Tolerx Inc. He has received consulting fees and/or honoraria for membership in advisory boards and honoraria for speaking from Amylin Pharmaceuticals, AstraZeneca, BerlinChemie/Menarini, Boehringer Ingelheim, Bristol-Myers Squibb, Diartis Pharmaceuticals, Eli Lilly \& Co., Hoffmann-LaRoche, GlaxoSmithKline, Intarcia Therapeutics, MannKind Corp., Merck Sharp \& Dohme, Novartis Pharma, NovoNordisk, Sanofi, Takeda and Wyeth Research, including reimbursement for travel expenses in connection with the above-mentioned activities. He owns no stock and is employed by Diabeteszentrum Bad Lauterberg, Germany. OB has no conflicts of interest to declare. RAR has received speaker or advisory board fees from AstraZeneca, Bristol-Myers Squibb, Eli Lilly \& Co., Merck Sharp \& Dohme, Novo Nordisk, Sanofi and Takeda. JJM has received lecture or advisory board fees from Astra Zeneca, Berlin-Chemie, Boehringer Ingelheim, BMS, Eli Lilly, Merck Sharp \& Dohme, NovoNordisk, Novartis, Roche and Sanofi-Aventis and research support from NovoNordisk, Merck Sharp \& Dohme, Sanofi-Aventis, Eli Lilly and Novartis.

Author contributions MAN and OB have screened the literature, extracted and analysed the data, and written the manuscript. RR and JJM were involved in conceptualising the analysis, critically discussing the results and reviewing the manuscript. All authors take 
responsibility for the content of this manuscript and have approved the final version for publication.

\section{References}

1. Drucker DJ, Nauck MA (2006) The incretin system: glucagon-like peptide-1 receptor agonists and dipeptidyl peptidase-4 inhibitors in type 2 diabetes. Lancet 368:1696-1705

2. Nauck MA, Kleine N, Ørskov C, Holst JJ, Willms B, Creutzfeldt W (1993) Normalization of fasting hyperglycaemia by exogenous glucagon-like peptide 1 (7-36 amide) in type 2 (non-insulin-dependent) diabetic patients. Diabetologia 36:741-744

3. Nauck MA, Weber I, Bach I et al (1998) Normalization of fasting glycaemia by intravenous GLP-1 ([7-36 amide] or [7-37]) in type 2-diabetic patients. Diabet Med 15:937-945

4. Nauck MA, Sauerwald A, Ritzel R, Holst JJ, Schmiegel W (1998) Influence of glucagon-like peptide 1 on fasting glycemia in type 2 diabetic patients treated with insulin after sulfonylurea secondary failure. Diabetes Care 21:1925-1931

5. Toft-Nielsen MB, Madsbad S, Holst JJ (2001) Determinants of the effectiveness of glucagon-like peptide- 1 in type 2 diabetes. J Clin Endocrinol Metab 86:3853-3860

6. Meier JJ, Gallwitz B, Salmen S et al (2003) Normalization of glucose concentrations and deceleration of gastric emptying after solid meals during intravenous glucagon-like peptide 1 in patients with type 2 diabetes. J Clin Endocrinol Metab 88:2719-2725

7. Nauck MA, Meier JJ (2005) Glucagon-like peptide 1 (GLP-1) and its derivatives in the treatment of diabetes. Regul Pept 124 (Suppl):135-148

8. Deacon CF, Nauck MA, Toft-Nielsen M, Pridal L, Willms B, Host JJ (1995) Both subcutaneously and intravenously administered glucagon-like peptide 1 are rapidly degraded from the $\mathrm{NH}_{2}$-terminus in type 2-diabetic patients and in healthy subjects. Diabetes 44:1126-1131

9. Deacon CF, Pridal L, Klarskov L, Olesen M, Holst JJ (1996) Glucagon-like peptide 1 undergoes differential tissue-specific metabolism in the anesthetized pig. Am J Physiol Endocrinol Metab 271:E458-E464

10. Nauck MA, Wollschläger D, Werner J et al (1996) Effects of subcutaneous glucagon-like peptide 1 (GLP-1 [7-36 amide]) in patients with NIDDM. Diabetologia 39:1546-1553

11. Ritzel R, Ørskov C, Holst JJ, Nauck MA (1995) Pharmacokinetic, insulinotropic, and glucagonostatic properties of GLP-1 [7-36 amide] after subcutaneous injection in healthy volunteers. Dose-responserelationships. Diabetologia 38:720-725

12. Toft-Nielsen M-B, Madsbad S, Holst JJ (1999) Continuous subcutaneous infusion of glucagon-like peptide 1 lowers plasma glucose and reduces appetite in type 2 diabetic patients. Diabetes Care 22:1137-1143

13. Zander M, Taskiran M, Toft-Nielsen MB, Madsbad S, Holst JJ (2001) Additive glucose-lowering effects of glucagon-like peptide1 and metformin in type 2 diabetes. Diabetes Care 24:720-725

14. Zander M, Madsbad S, Madsen JL, Holst JJ (2002) Effect of 6-week course of glucagon-like peptide 1 on glycaemic control, insulin sensitivity, and beta-cell function in type 2 diabetes: a parallelgroup study. Lancet 359:824-830

15. Zander M, Christiansen A, Madsbad S, Holst JJ (2004) Additive effects of glucagon-like peptide 1 and pioglitazone in patients with type 2 diabetes. Diabetes Care 27:1910-1914

16. Linnebjerg H, Kothare PA, Skrivanek $Z$ et al (2006) Exenatide: effect of injection time on postprandial glucose in patients with type 2 diabetes. Diabet Med 23:240-245
17. Kolterman OG, Kim DD, Shen L et al (2005) Pharmacokinetics, pharmacodynamics, and safety of exenatide in patients with type 2 diabetes mellitus. Am J Health Syst Pharm 62:173-181

18. Fineman MS, Bicsak TA, Shen LZ et al (2003) Effect on glycemic control of exenatide (synthetic exendin-4) additive to existing metformin and/or sulfonylurea treatment in patients with type 2 diabetes. Diabetes Care 26:2370-2377

19. Fineman MS, Shen LZ, Taylor K, Kim DD, Baron AD (2004) Effectiveness of progressive dose-escalation of exenatide (exendin-4) in reducing dose-limiting side effects in subjects with type 2 diabetes. Diabetes Metab Res Rev 20:411-417

20. Moretto TJ, Milton DR, Ridge TD et al (2008) Efficacy and tolerability of exenatide monotherapy over 24 weeks in antidiabetic drug-naive patients with type 2 diabetes: a randomized, doubleblind, placebo-controlled, parallel-group study. Clin Ther 30:14481460

21. DeFronzo RA, Ratner RE, Han J, Kim DD, Fineman MS, Baron AD (2005) Effects of exenatide (exendin-4) on glycemic control and weight over 30 weeks in metformin-treated patients with type 2 diabetes. Diabetes Care 28:1092-1100

22. Fehse F, Trautmann M, Holst JJ et al (2005) Exenatide augments first- and second-phase insulin secretion in response to intravenous glucose in subjects with type 2 diabetes. J Clin Endocrinol Metab 90:5991-5997

23. Taylor K, Kim D, Nielsen LL, Aisporna M, Baron AD, Fineman MS (2005) Day-long subcutaneous infusion of exenatide lowers glycemia in patients with type 2 diabetes. Horm Metab Res 37:627-632

24. Nauck MA, Hompesch M, Filipczak R, Le TD, Zdravkovic M, Gumprecht J (2006) Five weeks of treatment with the GLP-1 analogue liraglutide improves glycaemic control and lowers body weight in subjects with type 2 diabetes. Exp Clin Endocrinol Diabetes : Off J German Soc Endocrinol German Diabetes Assoc 114:417-423

25. Garber A, Henry R, Ratner R et al (2009) Liraglutide versus glimepiride monotherapy for type 2 diabetes (LEAD-3 Mono): a randomised, 52-week, phase III, double-blind, parallel-treatment trial. Lancet 373:473-481

26. Nauck M, Frid A, Hermansen K et al (2009) Efficacy and safety comparison of liraglutide, glimepiride, and placebo, all in combination with metformin, in type 2 diabetes: the LEAD (liraglutide effect and action in diabetes)-2 study. Diabetes Care 32:84-90

27. Buse JB, Rosenstock J, Sesti G et al (2009) Liraglutide once a day versus exenatide twice a day for type 2 diabetes: a 26-week randomised, parallel-group, multinational, open-label trial (LEAD-6). Lancet 374:39-47

28. Giordano A, Frontini A, Cinti S (2008) Adipose organ nerves revealed by immunohistochemistry. Methods Mol Biol 456:83-95

29. Hellström PM (2009) GLP-1: broadening the incretin concept to involve gut motility. Regul Pept 156:9-12

30. Schirra J, Nicolaus M, Woerle HJ, Struckmeier C, Katschinski M, Göke B (2009) GLP-1 regulates gastroduodenal motility involving cholinergic pathways. Neurogastroenterol Motil 21(609618):e21-e22

31. Falkén Y, Hellström PM, Holst JJ, Näslund E (2011) Changes in glucose homeostasis after Roux-en-Y gastric bypass surgery for obesity at day three, two months, and one year after surgery: role of gut peptides. J Clin Endocrinol Metab 96:2227-2235

32. Goldstein BJ, Feinglos MN, Lunceford JK, Johnson J, WilliamsHerman DE (2007) Effect of initial combination therapy with sitagliptin, a dipeptidyl peptidase-4 inhibitor, and metformin on glycemic control in patients with type 2 diabetes. Diabetes Care 30:1979-1987

33. Laferrere B, Heshka S, Wang K et al (2007) Incretin levels and effect are markedly enhanced 1 month after Roux-en-Y gastric bypass surgery in obese patients with type 2 diabetes. Diabetes Care 30:1709-1716 